\title{
The Effect of Tooth Extraction on Skeletal Open Bite Correctionin Adults: A Systematic Review
}

\section{Karim Mahmoud Awad ${ }^{1 *}$, Amr Abou Elezz ${ }^{2}$, Mohamed Ashraf Badawi ${ }^{3}$, Fady Hussein Fahim ${ }^{4}$ and Hend Salah ElSayed ${ }^{5}$}

${ }^{1}$ Associate Lecturer, Department of Orthodontics, Faculty of Oral and Dental

Medicine, Misr International University, Egypt

${ }^{2}$ Dean and Professor of Orthodontics, Faculty of Oral and Dental Medicine, Cairo

University, Egypt

${ }^{3} B D S$, MOrth RCSEd, MS Orthodontics, Faculty of Oral and Dental Medicine, Cairo

University, Egypt

${ }^{4}$ Lecturer, Department of Orthodontics, Faculty of Oral and Dental Medicine, Cairo

University, Egypt

${ }^{5}$ Researcher, Department of Orthodontics and Pediatric Dentistry, Oro-dental

Division, National Research Centre, Cairo, Egypt

*Corresponding Author: Karim Mahmoud Awad, Teaching Assistant, Department of Orthodontics, Faculty of Oral and Dental Medicine, Misr International University, Cairo, Egypt.
Received: October 01, 2021

Published: October 25, 2021

(C) All rights are reserved by Karim

Mahmoud Awad., et al.

\begin{abstract}
Objective: The aim of this systematic review is to summarize the available evidence regarding the effect of dental extraction on the facial vertical dimension in adults.

Materials and Methods: A health sciences librarian was consulted and a search strategy was developed and performed in February 2021 for electronic searches in MEDLINE/Pub Med, EMBASE, the Web of Science, the Cochrane Library and LILACS. Hand searching was performed in some of the major journals in the field and reference lists were also assessed. Two independent reviewers selected the studies to pre-specified eligibility criteria and extracted the data.

Results: The full texts of twenty articles were assessed. None of the articles met all the criteria of inclusion. All the excluded studies showed high risk of bias, due to their study design and lack of control for confounding factors such as growth, treatment mechanics and space deficiency.

Conclusion: There is no reliable evidence in the available literature that dental extraction versus non-extraction can decrease the facial vertical dimension in adults with skeletal open-bite. High quality controlled trials are needed to evaluate the effect of extraction.

Keywords: Evidence-Based Orthodontics; Cephalometrics; Extraction Vs. Nonextraction; Adult Treatment; Tooth Extraction; Vertical Dimension
\end{abstract}

\section{Introduction}

\section{Rationale}

A persistent challenge to orthodontists is the problem of skeletal open bite or increased facial vertical dimension. The goal of orthodontic treatment in these cases is to establish esthetics and facial balance as well as functional occlusion. The success and stability of treatment depend on identifying the underlying etiology and using proper mechanics to address them [1].

Citation: Karim Mahmoud Awad., et al. "The Effect of Tooth Extraction on Skeletal Open Bite Correction in Adults: A Systematic Review". Acta Scientific Dental Sciences 5.11 (2021): 71-78. 
Skeletal open bite is also referred to as vertical skeletal dysplasia or long and hyperdivergent faces. This is characterized by a steep mandibular plane angle, divergent facial patterns with increased anterior facial height, shortened posterior facial height [2] and decreased percentage of upper to lower facial height [3]. This increase in facial vertical dimension may or may not be accompanied by an anterior dental open bite, which is determined by the relationship between the upper and lower incisors. In skeletal hyperdivergence, anterior dental open bite is common and may extend to the posterior region in severe cases. However, deep bite may be present in $1 / 3$ of hyperdivergent cases [4].

Development of skeletal and dental open bite depends on the interaction of multiple determinants. The growth pattern (extent and direction) of the mandibular condyles, maxillary downward movement (extent and cant), ratio of anterior to posterior vertical growth and surface remodeling are important considerations in skeletal open bite [5]. Abnormal functional habits may cause dental and if persistent in young individuals, skeletal open bite [6]. Another key determinant of open bite is the amount and direction of the eruption of anterior and posterior teeth [7]. In skeletal open bite incisal over eruption may mask the discrepancy. On the other hand excessive over eruption of posterior teeth may lead to backward mandibular rotation and an anterior dental open bite or it may exaggerate an existing skeletal problem [8].

In growing patients, eliminating functional habits, restricting unfavorable growth patterns and/or enhancing dentoalveolar compensation may correct a skeletal or dental open bite [9]. While in adults, surgical interventions or dental compensation are the only options. Surgical procedures may include segmented or one-piece maxillary impaction, sagittal split osteotomies or a combination of these modalities [10]. Surgical interventions have been successful, with bi-maxillary surgeries showing less stability than single arch surgeries [11]. Dental compensation is commonly achieved by molar intrusion [12], incisal extrusion or a combination of these [13].

Dental camouflage has also been achieved through premolar extraction. The drawbridge effect where premolars are extracted and anterior teeth are retracted and tipped back has been used to close anterior open bite [14]. There is also a hypothesis that the elimination of the "wedge effect", in which the molar teeth are moved forward into the extraction spaces of the premolars, without extrusion, can cause the mandible to rotate forward decreasing the vertical facial dimension and the mandibular plane angle [15]. Another option is the extraction of the first or second molars to decrease the vertical dimension by eliminating the "wedge effect". Authors have claimed that such extractions reduce the hyper-divergence of the face $[16,17]$. Yet others do not support this hypothesis $[18,19]$.
Recent studies evaluating the effect of premolar extraction showed that vertical changes were not statistically different from those in non-extraction patients $[20,21]$.

\section{Objectives of the Study}

This systematic review was undertaken to summarize the best evidence on the effect of dental extraction on the skeletal vertical dimension in adults and to evaluate the need for further clinical trials. The PICO question was; in adult patients with increased vertical dimension (Skeletal open bite), will orthodontic dental extraction compared to non-extraction affect the vertical dimension?

\section{Materials and Methods}

\section{Protocol and registration}

A detailed a priori protocol was designed. The protocol was registered with PROSPERO (International prospective register of systematic reviews) the Centre for Reviews and Dissemination (CRD) (Registration number: 42013005755) [22].

\section{Search strategy}

An attempt was made to identify all eligible studies for this review. A health sciences librarian was consulted, and electronic searches until February 2021 were performed in MEDLINE (from 1946), EMBASE (from 1947), the Web of Science (from 1900), Cochrane Oral Health Group Trials Register, the Cochrane Central Register of Controlled Trials (CENTRAL) and LILACS (from 1982). Two independent reviewers performed the electronic search. The search terms for MEDLINE/Pub Med are shown in table 1. Hand searching was performed in the major journals in the field: American Journal of Orthodontics and Dentofacial Orthopedics, Angle Orthodontist, Journal of Orthodontics, European Journal of Orthodontics and Australian Orthodontic Journal. Hand searching of reference lists was performed. Authors of studies requiring further clarification were contacted.

\begin{tabular}{|c|c|}
\hline$\# 1$ & $\begin{array}{c}\text { (hyperdivergent or hyper divergent or openbite* or open } \\
\text { bite* or long face or high angle* or adenoid face).mp. }\end{array}$ \\
\hline$\# 2$ & $\begin{array}{r}\text { (extract* or premolar* or pre molar* or bicuspid* or bi } \\
\text { cuspid* or molar* or remov*).mp }\end{array}$ \\
\hline$\# 3$ & $\begin{array}{c}\text { (vertical dimension or mandibular rotation* or forward } \\
\text { rotation* or cephalometr* or cephalo metr*).mp. }\end{array}$ \\
\hline$\# 4$ & 1 and 2 and 3 \\
\hline$\# 5$ & Open Bite/ \\
\hline$\# 6$ & exp Tooth Extraction/ \\
\hline$\# 7$ & 5 and 6 \\
\hline$\# 8$ & 4 or 7 \\
\hline
\end{tabular}

Table 1: Search strategy MEDLINE/Pub Med. 


\section{Study selection}

Two reviewers performed article selection independently. Any disagreement between the two was discussed and resolved by consensus. The pre-specified eligibility criteria were randomized and non-randomized controlled trials, studies with non-treatment control group and cohort studies provided that data from a comparison group was reported, articles evaluating adult subjects with excessive vertical dimension indicated for orthodontic treatment with extraction of permanent teeth. Only studies evaluating skeletal open-bite were considered. The outcome evaluated was the skeletal vertical dimension, measured on lateral cephalometric radiographs. Studies using vertical extrusive/intrusive mechanics such as headgear or mini-screws were excluded due to their reported effects on the vertical dimension.

\section{Data collection}

Two authors extracted data independently. The following was reported: Study; year of publication and author(s), Methods; study design, method of cephalometric analysis and duration of treatment, Participants; sample size, subject's age and gender and criteria of increased vertical dimension and open bite, Interventions; extracted teeth, number of teeth extracted, and types of orthodontic mechanics used, and Outcomes: Frankfurt mandibular angle (FMA).

\section{Results}

The search returned 1345 citations. 474 duplicates were removed leaving 871 potential articles for screening. 851 articles were irrelevant or did not meet the inclusion criteria at the title and/abstract screening. The full articles of the remaining 20 studies $(14,16-21,23-35)$ were assessed. No article fulfilled all the eligibility criteria. The 20 excluded articles and reasons for rejection are reported in table 2. A summary of the process of article exclusion is reported in the PRISMA flow diagram (Figure 1-3).

\section{Discussion}

It is a common assumption that eliminating the "wedge effect" by extraction of posterior teeth rotates the mandible forward, decreases vertical facial height and aids in the correction of open bite [15]. This systematic review was conducted to evaluate if dental extraction is an efficient treatment option for increased skeletal vertical dimension. An attempt was made to collate the available evidence that evaluates the effect of extraction on facial height.

None of 20 studies that were assessed in full text met the eligibility criteria. Causes of exclusion of these articles were the inappropriate selection of subjects' criteria, the lack of a control group,

\begin{tabular}{|c|c|c|c|c|c|}
\hline & Authors*/year & & & & \\
\hline 1 & Al-Nimiri 2006 [23] & & $\dagger$ & $\sqrt{ }$ & M \\
\hline 2 & Aras $2002[16]$ & & $\dagger$ & $\sqrt{ }$ & \\
\hline 3 & Chua., et al. 1993 [24] & $\neq$ & & & $\mathrm{M}$ \\
\hline 4 & Cusimano., et al. 1998 [25] & & $\dagger$ & $\sqrt{ }$ & \\
\hline 5 & De Freitas., et al. 2004 [26] & & $\dagger$ & $\sqrt{ }$ & M \\
\hline 6 & Gkantidis., et al. 2011 [21] & & $\dagger$ & & M \\
\hline 7 & Hans., et al. 2006 [27] & & $\dagger$ & & \\
\hline 8 & Hayasaki., et al. 2005 [28] & $\neq$ & & & M \\
\hline 9 & Jacobs., et al. 2012 [17] & & $\dagger$ & & M \\
\hline 10 & Janson., et al. 2006 [14] & & $\dagger$ & $\sqrt{ }$ & $\mathrm{M}$ \\
\hline 11 & Kim., et al. 2005 [29] & & $\dagger$ & $\sqrt{ }$ & \\
\hline 12 & Klapper., et al. 1992 [30] & $\neq$ & & & M \\
\hline 13 & Kocadereli 1999 [18] & $\neq$ & $\dagger$ & & \\
\hline 14 & Kojima., et al. 2009 [31] & & $\dagger$ & & M \\
\hline 15 & Kumari and Fida 2010 [20] & & $\dagger$ & & \\
\hline 16 & Paquette and Johnston 1992 [32] & $\neq$ & & & \\
\hline 17 & Sarisoy and Darendeliler 1999 [33] & & $\dagger$ & & M \\
\hline 18 & Sivakumar and Valiathan 2008 [34] & $\neq$ & & & \\
\hline 19 & Staggers 1994 [19] & $\ddagger$ & & & \\
\hline 20 & Yamaguchi and Nanda 1991 [35] & & $\dagger$ & $\sqrt{ }$ & M \\
\hline
\end{tabular}

Table 2: Excluded article and reasons of exclusion.

*: Authors listed alphabetically, $\ddagger$ : Included subjects are not facially hyperdivergent, $\uparrow$ : Not adults (growing subjects), $\sqrt{ }$ : Study does not include a control group, M: Extrusive or intrusive mechanics were used.

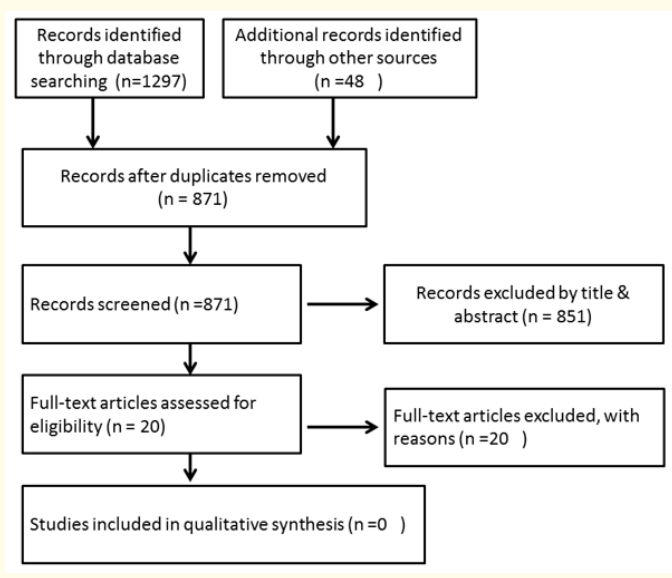

Figure 1: PRISMA flow chart of retrieved studies 


\begin{tabular}{|c|c|c|c|}
\hline Section/topic & \# & Checklist item & $\begin{array}{c}\text { Reported } \\
\text { on page } \\
\#\end{array}$ \\
\hline \multicolumn{4}{|l|}{ TITLE } \\
\hline Title & 1 & Identify the report as a systematic review, meta-analysis, or both. & 1 \\
\hline \multicolumn{4}{|l|}{ ABSTRACT } \\
\hline $\begin{array}{l}\text { Structured } \\
\text { summary }\end{array}$ & 2 & $\begin{array}{l}\text { Provide a structured summary including, as applicable: background; } \\
\text { objectives; data sources; study eligibility criteria, participants, and } \\
\text { interventions; study } \\
\text { appraisal and synthesis methods; results; limitations; conclusions and } \\
\text { implications of key findings; systematic review registration number. }\end{array}$ & 1 \\
\hline \multicolumn{4}{|l|}{ INTRODUCTION } \\
\hline Rationale & 3 & $\begin{array}{l}\text { Describe the rationale for the review in the context of what is already } \\
\text { known. }\end{array}$ & 1 \\
\hline Objectives & 4 & $\begin{array}{l}\text { Provide an explicit statement of questions being addressed with } \\
\text { reference to } \\
\text { participants, interventions, comparisons, outcomes, and study design } \\
\text { (PICOS). }\end{array}$ & 3 \\
\hline \multicolumn{4}{|l|}{ METHODS } \\
\hline $\begin{array}{l}\text { Protocol and } \\
\text { registration }\end{array}$ & 5 & $\begin{array}{l}\text { Indicate if a review protocol exists, if and where it can be accessed (e.g., } \\
\text { Web } \\
\text { address), and, if available, provide registration information including } \\
\text { registration number. }\end{array}$ & 3 \\
\hline $\begin{array}{l}\text { Eligibility } \\
\text { criteria }\end{array}$ & 6 & $\begin{array}{l}\text { Specify study characteristics (e.g., PICOS, length of follow-up) and report } \\
\text { characteristics (e.g., years considered, language, publication status) used } \\
\text { as } \\
\text { criteria for eligibility, giving rationale. }\end{array}$ & 3 \\
\hline $\begin{array}{l}\text { Information } \\
\text { sources }\end{array}$ & 7 & $\begin{array}{l}\text { Describe all information sources (e.g., databases with dates of coverage, } \\
\text { contact with study authors to identify additional studies) in the search } \\
\text { and date last searched. }\end{array}$ & 3 \\
\hline Search & 8 & $\begin{array}{l}\text { Present full electronic search strategy for at least one database, including } \\
\text { any limits used, such that it could be repeated. }\end{array}$ & 3 \\
\hline Study selection & 9 & $\begin{array}{l}\text { State the process for selecting studies (i.e., screening, eligibility, included } \\
\text { in } \\
\text { systematic review, and, if applicable, included in the meta-analysis). }\end{array}$ & 4 \\
\hline $\begin{array}{l}\text { Data collection } \\
\text { process }\end{array}$ & 10 & $\begin{array}{l}\text { Describe method of data extraction from reports (e.g., piloted forms, } \\
\text { independently, in duplicate) and any processes for obtaining and } \\
\text { confirming data from investigators. }\end{array}$ & 4 \\
\hline Data items & 11 & $\begin{array}{l}\text { List and define all variables for which data were sought (e.g., PICOS, } \\
\text { funding sources) and any assumptions and simplifications made. }\end{array}$ & 4 \\
\hline $\begin{array}{l}\text { Risk of bias in } \\
\text { individual } \\
\text { studies }\end{array}$ & 12 & $\begin{array}{l}\text { Describe methods used for assessing risk of bias of individual studies } \\
\text { (including specification of whether this was done at the study or outcome } \\
\text { level), and how this information is to be used in any data synthesis. }\end{array}$ & 4 \\
\hline $\begin{array}{l}\text { Summary } \\
\text { measures }\end{array}$ & 13 & $\begin{array}{l}\text { State the principal summary measures (e.g., risk ratio, difference in } \\
\text { means). }\end{array}$ & $\mathrm{N} / \mathrm{A}$ \\
\hline
\end{tabular}




\begin{tabular}{|c|c|c|c|}
\hline $\begin{array}{l}\text { Synthesis of } \\
\text { results }\end{array}$ & 14 & $\begin{array}{l}\text { Describe the methods of handling data and combining results of studies, } \\
\text { if done, including measures of consistency (e.g., }{ }^{2} \text { ) for each meta-analy- } \\
\text { sis. }\end{array}$ & $\mathrm{N} / \mathrm{A}$ \\
\hline Section/topic & $\#$ & Checklist item & $\begin{array}{l}\text { Reported } \\
\text { on page } \\
\#\end{array}$ \\
\hline $\begin{array}{l}\text { Risk of bias } \\
\text { across studies }\end{array}$ & 15 & $\begin{array}{l}\text { Specify any assessment of risk of bias that may affect the cumulative } \\
\text { evidence (e.g., publication bias, selective reporting within studies). }\end{array}$ & $\mathrm{N} / \mathrm{A}$ \\
\hline $\begin{array}{l}\text { Additional } \\
\text { analyses }\end{array}$ & 16 & $\begin{array}{l}\text { Describe methods of additional analyses (e.g., sensitivity or subgroup } \\
\text { analyses, meta-regression), if done, indicating which were pre-specified. }\end{array}$ & $\mathrm{N} / \mathrm{A}$ \\
\hline \multicolumn{4}{|l|}{ RESULTS } \\
\hline Study selection & 17 & $\begin{array}{l}\text { Give numbers of studies screened, assessed for eligibility, and included in } \\
\text { the review, with reasons for exclusions at each stage, ideally with a flow } \\
\text { diagram. }\end{array}$ & 4 \\
\hline $\begin{array}{l}\text { Study character- } \\
\text { istics }\end{array}$ & 18 & $\begin{array}{l}\text { For each study, present characteristics for which data were extracted } \\
\text { (e.g., study size, PICOS, follow-up period) and provide the citations. }\end{array}$ & $\mathrm{N} / \mathrm{A}$ \\
\hline $\begin{array}{l}\text { Risk of bias } \\
\text { within studies }\end{array}$ & 19 & $\begin{array}{l}\text { Present data on risk of bias of each study and, if available, any outcome } \\
\text { level assessment (see item 12). }\end{array}$ & $\mathrm{N} / \mathrm{A}$ \\
\hline $\begin{array}{l}\text { Results of indi- } \\
\text { vidual studies }\end{array}$ & 20 & $\begin{array}{l}\text { For all outcomes considered (benefits or harms), present, for each study: } \\
\text { (a) simple summary data for each intervention group (b) effect estimates } \\
\text { and confidence intervals, ideally with a forest plot. }\end{array}$ & $\mathrm{N} / \mathrm{A}$ \\
\hline $\begin{array}{l}\text { Synthesis of } \\
\text { results }\end{array}$ & 21 & $\begin{array}{l}\text { Present results of each meta-analysis done, including confidence inter- } \\
\text { vals and measures of consistency. }\end{array}$ & $\mathrm{N} / \mathrm{A}$ \\
\hline $\begin{array}{l}\text { Risk of bias } \\
\text { across studies }\end{array}$ & 22 & $\begin{array}{l}\text { Present results of any assessment of risk of bias across studies (see Item } \\
\text { 15). }\end{array}$ & $\mathrm{N} / \mathrm{A}$ \\
\hline $\begin{array}{l}\text { Additional } \\
\text { analysis }\end{array}$ & 23 & $\begin{array}{l}\text { Give results of additional analyses, if done (e.g., sensitivity or subgroup } \\
\text { analyses, meta-regression [see Item 16]). }\end{array}$ & $\mathrm{N} / \mathrm{A}$ \\
\hline \multicolumn{4}{|l|}{ DISCUSSION } \\
\hline $\begin{array}{l}\text { Summary of } \\
\text { evidence }\end{array}$ & 24 & $\begin{array}{l}\text { Summarize the main findings including the strength of evidence for each } \\
\text { main outcome; consider their relevance to key groups (e.g., healthcare } \\
\text { providers, users, and policy makers). }\end{array}$ & 5 \\
\hline Limitations & 25 & $\begin{array}{l}\text { Discuss limitations at study and outcome level (e.g., risk of bias), and at } \\
\text { review-level (e.g., incomplete retrieval of identified research, reporting } \\
\text { bias). }\end{array}$ & $5-8$ \\
\hline Conclusions & 26 & $\begin{array}{l}\text { Provide a general interpretation of the results in the context of other } \\
\text { evidence, and implications for future research. }\end{array}$ & 8 \\
\hline \multicolumn{4}{|l|}{ FUNDING } \\
\hline Funding & 27 & $\begin{array}{l}\text { Describe sources of funding for the systematic review and other support } \\
\text { (e.g., supply of data); role of funders for the systematic review. }\end{array}$ & \\
\hline
\end{tabular}

Table 3: PRISMA 2009 Checklist.

From: Moher D, Liberati A, Tetzlaff J, Altman DG, The PRISMA Group (2009). Preferred Reporting Items for Systematic Reviews and Meta-Analyses: The PRISMA Statement. PLoS Med 6(6): e1000097. doi:10.1371/journal.pmed1000097.

For more information, visit: www.prisma-statement.org. 
and confounding factors such as growth and treatment mechanics that affected the vertical dimension.

\section{Limitations of the excluded studies}

Study designs

A limitation of this review is that the search results included no randomized clinical trials (RCT). Randomization and allocation concealment was not applicable in all excluded studies leading to high risk of selection bias. Common problems related to retrospective cohort studies include the inherent problems of inadequate reporting, missing data, inadequate matching of subjects and control groups.

Different treatment mechanics were used in the intervention and control group $[19,21]$. There were even heterogeneity within the intervention group itself, where some subjects extracted first premolars while others extracted second premolars either maxillary or mandibular $[14,26]$. Similarly, in the study by Sarisoy and Darendeliler not all patients were prescribed a headgear and headgears were either high or low pull according to the growth pattern [33].

\section{Research methodology}

An important factor to consider in clinical trials is the selection of subjects in the control and the experimental group. Selected subjects must validly represent the targeted population. This review evaluated the extraction effect on hyper-divergent patients. Articles that included subjects with normal facial vertical dimension or other skeletal malocclusions were excluded [18,19,24,28,30,32,34].

The lack of a control group in the studies prevented a reliable assessment of the effect of the extraction. Therefore, in an attempt to include higher quality evidence, articles with no control groups were excluded $[14,16,23,25,26,29,35]$.

It was not clear if any attempt of blinding the clinicians and outcome assessors was made, producing an unclear risk of performance and detection bias respectively.

The sample sizes in all excluded studies were relatively small. None of the authors calculated the sample size required to detect significant difference between the compared groups at an adequate study power.

\section{Confounding factors and interactions}

Growth

The authors of the excluded studies assumed that the influence of growth is very limited in their sample. However, this is arguable since at least a portion of the subjects under investigation were of growing age [14,16,18,20,21,23,25-27,29,33,35]. The studies did not use a proper indicator for skeletal assessment to confirm the cessation of facial growth except two studies used the handwrist radiograph $[16,33]$ and one study used the cervical vertebrae maturation index [21]. The other authors reported only the chronological age of the participants, which is unreliable for assessing skeletal maturation [5].

\section{Crowding}

In cases with crowding or where incisal retraction is indicated, the extraction space is utilized for such purposes, preventing the mesial movement of the posterior teeth and anterior rotation of the mandible. In some of the excluded articles, the patient selection criteria was mild crowding to allow the forward movement of the posterior teeth $[16,17,21]$ while the other articles neglected this criterion. To test the wedge effect hypothesis, subjects should have minimum to no crowding.

\section{Treatment mechanics}

Treatment mechanics producing a vertical effect (intrusive or extrusive) will act as a confounding factor [21]. Therefore, articles having such mechanics were excluded [14,17,21,23,24,26,28,3 $0,31,33,35]$. The use of vertical elastics (diagonal, class I, class II $[16,21,23,30]$ or class III $[16,17])$, low-pull headgear $[21,30,33,35]$, tip-back mechanics and expansion appliances [14,26], all cause extrusion and increase the vertical dimension [19,21,30,31]. Extrusive mechanics must be used with caution especially in hyperdivergent cases with anterior open-bite. On the other hand, tweed mechanics, high-pull headgear [35], mini-screws and bite plates produce intrusion or control the vertical height and maintain or decrease the vertical dimension.

Reporting change in the vertical position of the posterior and anterior teeth would have helped to identify and explain if dental extrusion could have affected the facial vertical dimension and overbite. Some of the excluded articles reported such change [17$21,25,26,28,30,31,34,35]$. 
Inadequate reporting

In the excluded studies, due to inadequate reporting of the statistical analysis of the pre and post treatment values, we were unable to completely interpret the effect of extractions of first molars [27]. Studies did not report any information regarding ethical approval or obtaining the participant's informed consents [18,29,31,33,35]. Although this doesn't influence the interpretation of the results, it shows a lack of standard reporting.

\section{Findings of excluded studies}

The excluded studies evaluating the extraction of the four first premolars fail to show a reduction in the facial height $[20,21,25,33]$. Similar results were seen for the effect of second premolar extractions and first molar extractions [16,23,28,29]. Maxillary second molar extraction failed to show a decrease in facial height [31]. There is some evidence that mandibular second molar extraction, with vertical elastics, in patients with vertical growth patterns and moderate skeletal Class III, may increase overbite and overjet despite the increase in lower facial height [17]. Careful interpretation of these results is required as the evidence show high risk of bias. The study designs and the confounded methodology are not ideal for assessing the therapeutic effects of extraction on the vertical dimension and open bite in adult patients. Nevertheless, this data represents the available evidence to date and could serve as a starting point for future research.

\section{Summary of evidence}

There is an absence of randomized controlled trials (RCTs) evaluating the effect of dental extraction on vertical dimensions and anterior open bite. The available literature included controlled trials, observational studies and case reports that did not meet eligibility criteria of this review.

\section{Conclusion}

\section{Implications for clinical practice}

It is a common assumption that extraction of posterior teeth eliminates the wedge effect, rotates the mandible forward, decreases vertical facial height and aids in the correction of open bite. However there is no reliable evidence in the literature to support this practice.

\section{Implication for future research}

- High quality studies with proper selection criteria of patient and control groups, and controlled for cofounders, are needed.
- Vertical dimensional change should be evaluated through composite outcomes, not simply a single outcome.

- Standardization of reliable and valid research methodology may be valuable for future meta-analysis.

- $\quad$ The reporting quality of studies, on selection and diagnostic criteria of subjects, detailed treatment protocol and statistical results should be improved by adhering to the reporting guidelines to allow for better interpretation of the results.

\section{Bibliography}

1. Ngan P and Fields HW. "Open bite: a review of etiology and management”. Pediatric Dentistry 19 (1997): 91-98.

2. Cangialosi T J. "Skeletal morphologic features of anterior open bite". American Journal of Orthodontics 85 (1984): 28-36.

3. Fields HW., et al. "Facial pattern differences in long facial children and adults". American Journal of Orthodontics 85 (1984): 217-223.

4. Proffit WR., et al. "Contemporary treatment of dentofacial deformity". St. Louis, Mo: Mosby Elsevier 4th edition (2003).

5. Bjork A and Skieller V. "Facial development and tooth eruption: An implant study at the age of puberty". American Journal of Orthodontics and Dentofacial Orthopedics 62 (1972): 339383.

6. Mizrahi E. "A review of anterior open bite". British Journal of Orthodontics 5 (1978): 21-27.

7. Moyers RE and Moyers RE. "Handbook of Orthodontics". Chicago. Year Book Medical Publishers (1998).

8. Enlow DH and Hans MG. "Essentials of Facial Growth". Philadelphia: Saunders (1996).

9. Lentini-Oliveira DA., et al. "Orthodontic and orthopedic treatment for anterior open bite in children". Cochrane Database of Systematic Reviews 2 (2007): CD005515.

10. Hoppenreijs TJ., et al. "Skeletal and dento-alveolar stability of Le Fort I intrusion osteotomies and bimaxillary osteotomies in anterior open bite deformities. A retrospective three centre study". International Journal of Oral and Maxillofacial Surgery 26 (1997): 161-175.

11. Greenlee MG., et al. "Stability of treatment for anterior openbite malocclusion: A meta-analysis". American Journal of Orthodontics and Dentofacial Orthopedics 139 (2011): 154-169. 
12. Ng J., et al. "True molar intrusion attained during orthodontic treatment: A systematic review". American Journal of Orthodontics and Dentofacial Orthopedics 130 (2006):709-714.

13. Kucukkeles N., et al. "Cephalometric evaluation of open bite treatment with NTi arch wires and anterior elastics". American Journal of Orthodontics and Dentofacial Orthopedics 116 (1999): 555-562.

14. Janson G., et al. "Stability of anterior open-bite extraction and nonextraction treatment in the permanent dentition". American Journal of Orthodontics and Dentofacial Orthopedics 129 (2006): 768-774.

15. Schudy FF. "The control of vertical overbite in clinical orthodontics". The Angle Orthodontist 38 (1968): 19-39.

16. Aras A. "Vertical changes following orthodontic extraction treatment in skeletal open bite subjects". European Journal of Orthodontics 4 (2002): 407-416.

17. Jacobs C., et al. "Dental compensation for moderate Class III with vertical growth pattern by extraction of the lower second molars". The Journal of Orofacial Orthopedics 73 (2012): 41-48.

18. Kocadereli I. "The effect of first premolar extraction on vertical dimension". American Journal of Orthodontics and Dentofacial Orthopedics 116 (1999): 41-45.

19. Staggers JA. "Vertical changes following first premolar extractions". American Journal of Orthodontics and Dentofacial Orthopedics 105 (1994): 19-24.

20. Kumari M and Fida M. "Vertical facial and dental arch dimensional changes in extraction vs. non-extraction orthodontic treatment". Journal of the College of Physicians and Surgeons Pakistan 20 (2010): 17-21.

21. Gkantidis N., et al. "Treatment strategies for patients with hyperdivergent Class II Division I malocclusion, is vertical dimension affected?" American Journal of Orthodontics and Dentofacial Orthopedics 140 (2011): 346-355.

22. Awad K. "The effect of tooth extraction choice on open bite correction: A Systematic Review" (2017).

23. Al Nimri KS. "Vertical changes in Class II Division I malocclusion after premolar extractions". The Angle Orthodontist 76 (2006): 52-58.

24. Chua AL., et al. "The effects of extraction versus nonextraction orthodontic treatment on the growth of the lower anterior face height". American Journal of Orthodontics and Dentofacial Orthopedics 104 (1993): 361-368.

25. Cusimano C., et al. "Effects of first bicuspid extraction on facial height in high-angle cases". Journal of Clinical Orthodontics 27 (1993): 594-598.
26. De Freitas M., et al. "Long-term stability of anterior open bite extraction treatment in the permanent dentition". American Journal of Orthodontics and Dentofacial Orthopedics 125 (2004): 78-87.

27. Hans M., et al. "Cephalometric changes in overbite and vertical facial height after removal of 4 first molars or first premolars". American Journal of Orthodontics and Dentofacial Orthopedics 130 (2006): 183-188.

28. Hayasaki S., et al. "Influence of extraction and non-extraction orthodontic treatment in Japanese-Brazilians with Class I and Class II Division I malocclusion". American Journal of Orthodontics and Dentofacial Orthopedics 127 (2005): 30-36.

29. Kim T., et al. "First or second premolar extraction effects on facial vertical dimension". The Angle Orthodontist 75 (2005): 177-182.

30. Klapper L., et al. "The influence of extraction and non-extraction orthodontic treatment on brachyfacial and dolichofacial growth patterns". American Journal of Orthodontics and Dentofacial Orthopedics 101 (1992): 425-430.

31. Kojima K., et al. "Effects of maxillary second molar extraction on dentofacial morphology before and after anterior openbite treatment: a Cephalometric study". Odontology 97 (2009): 43-50.

32. Paquette D and Johnston L. "A long-term comparison of nonextraction and premolar extraction edgewise therapy in "borderline" Class II patients". American Journal of Orthodontics and Dentofacial Orthopedics 102 (1992): 1-14.

33. Sarisoy L and Darendeliler N. "The influence of extraction orthodontic treatment on craniofacial structures: evaluation according to two different factors". American Journal of Orthodontics and Dentofacial Orthopedics 115 (1999): 508-514.

34. Sivakumar A and Valiathan A. "Cephalometric assessment of dentofacial vertical changes in Class I subjects treated with and without extractions". American Journal of Orthodontics and Dentofacial Orthopedics 133 (2008): 869-875.

35. Yamaguchi $\mathrm{K}$ and Nanda R. "The effects of extraction and nonextraction treatment on the mandibular-position". American Journal of Orthodontics and Dentofacial Orthopedics 100 (1991): 443-452.

\section{Volume 5 Issue 11 November 2021 (C)All rights are reserved by Karim Mahmoud Awad.,} et al. 